Audiology

Neurotology
Audiol Neurotol 2020;25:50-59

DOI: $10.1159 / 000502252$
Received: June 6, 2019

Accepted: July 19, 2019

Published online: September 10, 2019

\title{
Early Assessment of Vestibular Function after Unilateral Cochlear Implant Surgery
}

\author{
Maurizio Barbara Rita Talamonti Anna Teresa Benincasa Silvia Tarentini \\ Chiara Filippi Edoardo Covelli Simonetta Monini \\ Otolaryngology Unit, Department of Clinical and Molecular Medicine, Sapienza University Hospital Sant'Andrea, \\ Rome, Italy; NESMOS Department, Sapienza University Hospital Sant'Andrea, Rome, Italy
}

\section{Keywords}

Cochlear implantation · Vertigo $\cdot$ Balance disorders $\cdot$ Round window approach

\begin{abstract}
Introduction: Cochlear implantation $(\mathrm{Cl})$ has been reported to negatively affect vestibular function. The study of vestibular function has variably been conducted using different types of diagnostic tools. The combined use of modern, rapidly performing diagnostic tools could prove useful for standardization of the evaluation protocol. Methods: In a group of 28 subjects undergoing $\mathrm{Cl}$, the video head impulse test (vHIT), the cervical vestibular evoked myogenic potentials (cVEMP) and the short form of the Dizziness Handicap Inventory $(\mathrm{DHI})$ questionnaire were investigated preoperatively and postoperatively (implant on and off) in both the implanted and the contralateral, nonimplanted ear. All surgeries were performed with a round window approach (RWA), except for 3 otosclerosis cases in which the extended RWA (eRWA) was used. Results: The vHIT of the lateral semicircular canal showed preoperative vestibular involvement in nearly $50 \%$ of the cases, while the 3 canals were contemporarily affected in only $14 \%$ of the cases. In all the hypofunctional subjects, cVEMP were absent. A low VOR gain in all of the investigated superior semicircular canals was found in 4
\end{abstract}

subjects (14\%). In those subjects $(21.7 \%)$ in whom cVEMP were preoperatively present and normal on the operated side, the absence of a response was postoperatively recorded. Discussion/Conclusion: The vestibular protocol applied in this study was found to be appropriate for distinguishing between the $\mathrm{Cl}$-operated ear and the nonoperated ear. In this regard, cVEMP was found to be more sensitive than VHIT for revealing a vestibular sufferance after $\mathrm{Cl}$, though without statistical significance. Finally, the use of RWA surgery apparently did not reduce the occurrence of signs of vestibular impairment.

(C) 2019 The Author(s) Published by S. Karger AG, Basel

\section{Introduction}

The entirety of membranous and fluid components of the inner ear lies encased within the otic capsule (OC) that acts as a protective shield from external noxious agents. While sharing this space, the 2 labyrinthine components, i.e., the anteriorly located (cochlear) one and the posterior one (vestibular), are to some extent potentially involved in traumatic or pathological issues, even if only 1 of the 2 in the primarily offended. This relationship appears evident, for example, in the numerous histopathological reports showing ultrastructural changes in both 
compartments in the presence of an inner ear disorder (Ménière's disease, congenital malformations, labyrinthitis, etc.).

Cochlear implantation (CI) currently represents the best choice for recovering communication abilities in individuals with a poor hearing performance and encompasses the placement of a neural stimulator inside the cochlea, thereby violating the OC anatomical barrier. On one hand, this traumatic act allows the most appropriate electric stimulation of the auditory nerve fibers with hearing advantages; on the other hand, it could theoretically affect the more or less adjacently located vestibular end organs.

The possibility of an ensuing vestibular issue after CI has long been described and it has been debated since the early 1970s [Black, 1977]. In particular, a vestibular dysfunction can be expected in 3.1-77\% of CI subjects [Kubo et al., 2001; Melvin et al., 2009]; an improvement at activation has also been reported [Eisenberg et al., 1982; Buchman et al., 2004; Basta et al., 2008; Jin et al., 2008; Coordes et al., 2012; Parkes et al., 2017], while subjective dizziness occurs in 2-49\% of cases regardless of the severity of dysfunction [Batuecas-Caletrio et al., 2015]. The reason for such variability is likely related to the different timings of evaluations (short or long term after surgery) but mostly to the methodology for studying vestibular function which, in the last decade, has been implemented via objective diagnostic procedures (video head impulse test [vHIT] and vestibular evoked myogenic potentials [VEMP]) that have joined or progressively replaced at many Institutions the classical vestibular assessment setting of caloric and rotatory chair tests. Among the advantages of using the above mentioned tools is the possibility of obtaining specific information from a single vestibular receptor: the 3 semicircular canals (SSC) via the vHIT, the utricle via the ocular VEMP, and the saccule via the cervical VEMP (cVEMP).

At our Institution, a prospective evaluation study was conducted with the aim of highlighting the occurrence and incidence of vestibular impairment in an adult CI population at the time of its activation. For this purpose, the vHIT and cVEMP outcomes were merged with the subjective evaluation collected using the Dizziness Handicap Inventory (DHI) questionnaire.

\section{Materials and Methods}

A consecutive group of CI candidates was prospectively selected for the present study. Patients with other pathologies that could directly affect postural control, such as central nervous system dis- eases or orthopedic diseases, were excluded. All patients underwent implantation with the electrodes preferably inserted via the transmastoid facial recess surgery using the round window approach (RWA), performed by the same surgeon (M.B.). When the anatomy of the round window niche was distorted by ossification, the extended round window niche approach (eRWA) was used as an alternative [Roland et al., 2007]. Each implanted electrode reached full insertion in a single pass without any resistance or complication and the auditory nerve response telemetry was obtained in all of the patients.

According to our present protocol for studying vestibular disorders, the vestibule-ocular reflex (VOR) and the cVEMP were examined in each patient on both sides before surgery and after activation (around 1 month after surgery), with the implant switched on and off; the latter was merely for exploring the effects of surgery. The simplified DHI questionnaire was also administered before surgery and 1 month after CI.

\section{Vestibulo-Ocular Reflex}

The VOR was evaluated with the head-impulse test (HIT) for all 3 SSC, though a constant response could not be obtained in all of the tested subjects, with lateral SSC (LSC) vHIT showing more consistency with respect to SSC and posterior SSC (PSC). Therefore, when reporting data, the number of reliably assessed subjects out of the whole study cohort will be specified (in parentheses). During the test, the examiner/performer stood behind the patient, grasping the patient's head firmly with both hands. The patient was asked to keep looking at a stationary object at a distance of $90-100 \mathrm{~cm}$ from the wall. The head was then quickly and unpredictably turned through $10-20^{\circ}$ on the horizontal plane to the left or to the right, which permits testing of the corresponding LSC. Likewise, the same movements were performed on the plane of the SSC and the PSC. In order to register and measure the head and eye velocity during the head impulse, a video HIT system (vHIT; ICS Impulse System, GN Otometrics, Taastrup, Denmark) was used. The patient wore a pair of lightweight, tightly fitting goggles with a small video camera and a half-silvered mirror that reflected the image of the patient's right eye into the camera. The eye was illuminated by a low-level infrared light-emitting diode, and a small sensor on the goggles measured the head movement. The whole goggle system weighs about $60 \mathrm{~g}$ and was secured tightly to the head to minimize slippage. After calibration, the procedure began.

The head movement speed is measured by the sensor in the goggles, and the image of the eye is captured by the high-speed camera $(250 \mathrm{~Hz})$ and processed to yield the eye velocity. At the end of each head turn, the head velocity stimulus and the eye velocity response are displayed simultaneously on the screen so that the clinician can see whether the stimulus and the response were adequate, providing a quick way to maximize the quality of the head impulse. The software itself provides an algorithm that automatically rejects possible artefacts to limit subjective interpretations of tracings. Under normal conditions (healthy patients), the physician should observe that the patient is able to maintain the eyes fixed on the stationary target during the high-speed head rotation. Should a unilateral vestibular weakness exist, the eyes first drift in the same direction as the head and then compensatory refixation saccades are used to reset the visual fixation on the target. In a full test, 20 impulses were delivered randomly in each direction and, at the end, all head velocity stimuli and eye veloc- 
ity responses were displayed on the screen, allowing evaluation of the mean VOR gain (ratio of eye velocity to head velocity) for every head rotation and the appearance of saccades (covert or overt catch-up saccades) after head impulses to the right and to the left. Absolute values of VOR gain $>0.8$ were considered normal [Alhabib and Saliba, 2017], and values $<0.8$ were considered abnormal and a sign of hypofunction. Also, the appearance of covert or overt saccades in the postoperative vHIT test was considered abnormal.

\section{Vestibular Evoked Myogenic Potentials}

This examination was also performed in all subjects before CI on both the implanted side and the nonimplanted side. The cVEMP were searched with the aim of obtaining evidence of the functional condition of the saccule, using Eclipse Lediso software (Interacustic A/S, Assens, Denmark) in a soundproof room. A recording electrode was placed on the junction between the middle and upper one third of the sternocleidomastoid muscle on each side, while the reference electrode was placed on the sternoclavicular joint and the ground electrode was placed on the forehead. During the recording, the patient was instructed to sit in an upright position and turn their head sideways $45^{\circ}$ to generate a constant tonic pretension of the sternocleidomastoid muscle. A resistance of $<5 \mathrm{k} \Omega$ was required for each electrode. The acoustic stimuli (short tone bursts: 1/4; $95 \mathrm{~dB} \mathrm{HL}$ and $500 \mathrm{~Hz}$; rate: 1/4; 5.1/s; ramp: $1 / 4 ; 1 \mathrm{~ms}$; and plateau: $1 / 4 ; 2 \mathrm{~ms}$ ) were delivered monaurally through a headphone (air conduction or AC), and the myogenic potential was recorded ipsilaterally using surface electrodes. The analysis time was $70 \mathrm{~ms}$, and the electromyographic signal was band-pass filtered from 1 to 2,000 Hz. Every set of 100 stimuli was averaged and repeated twice to verify reproducibility. The test was performed in both the implanted ear and the nonimplanted ear pre- and postoperatively. If there were no recognizable or reproducible waveforms, the cVEMP response was considered absent $(\mathrm{A})$; when present $(\mathrm{P})$, an abnormal or normal response was defined by looking at the p13 (P1)n23 (N1) latency and amplitude peaks, without considering the relative thresholds.

\section{Dizziness Handicap Inventory}

A short form (DHIsf) of the Italian version of the DHI questionnaire was used [Nola et al., 2010]. This questionnaire is internationally renowned as a valid tool for collecting individual clini$\mathrm{cal}$ data on vestibular impairment. The retained items explore the domains of eye/head movements, full body activities, and mood alterations. The physician explains the aim of the questionnaire, and the patient fills out the questionnaire. The questionnaires were answered in a similar fashion to the original English version. The patient had to answer "yes," "sometimes," or "no" to each question, with the responses being given a value of 4,2 , or 0 , respectively. The questionnaire has 25 items; thus, the total score could range from 0 to 100. A change in the total DHI score of $>6$ points after implantation was considered significant. The patients were also invited to refer the eventual presence of vertigo both pre- and postoperatively.

\section{Statistical Analysis}

Statistical analyses were performed using SPSS version 23.0 (IBM Corp.; USA). The comparison was carried out separately for implanted and nonimplanted ears and for each time of evaluation
Table 1. Etiology of deafness in the study group

\begin{tabular}{lc}
\hline Etiology & Cases, $n$ \\
\hline Otosclerosis & 7 \\
Chronic otitis & 3 \\
Ménière (drop attacks) & 1 \\
Progressive SNHL & 13 \\
Ototoxicity & 1 \\
Meningitis & 1 \\
Temporal bone fracture & 1 \\
Congenital & 1 \\
\hline
\end{tabular}

(preoperatively and post-operatively with the implant off and on). vHIT were analyzed using ANOVA, cVEMPS were compared using the McNemar test, and a paired $t$ test was applied when comparing DHI values in pre- and postoperative conditions. In all analyses, $p<0.05$ was considered statistically significant.

\section{Results}

A total of 28 subjects ( 11 females and 17 males) aged 19-83 years (mean 57.4) were enrolled into this study and it was conducted at a tertiary university hospital. The study protocol was approved by the local institutional review board. According to bilateral hearing impairment and, mostly, to the poor speech discrimination and the insufficient performance when aided by last-generation powerful conventional hearing aids, they were all candidates for CI, which was always performed unilaterally. Only 1 subject was already wearing a CI on the other ear, so she was the only bilateral CI in the present study. The etiology of deafness is shown in Table 1. Patients were all implanted with Synchrony ${ }^{\circledR}$ device (Medel, Innsbruck, Austria), except for 2 patients who received a CI522 Nucleus ${ }^{\circledR}$ device (Cochlear, Melbourne, VIC, Australia). In only 1 subject with partial deafness was a hearing preservation protocol adopted; it included intravenous administration of steroids the day before surgery, the Softflex ${ }^{\circledR}$ device via the RWA, and intraoperative application of gelfoamsoaked steroid pledgets. The planned insertion site (round window membrane approach or RWA) was, however, not practicable in 3 subjects who presented extensive otosclerotic ossification in that area and in whom the eRWA was applied.

The preoperative vestibular function revealed the following findings. 
Fig. 1. vHIT for the lateral SSC in preoperative and postoperative conditions (in off and on situations).
Fig. 2. vHIT for the superior SSC in preoperative and postoperative conditions (in off and on situations).
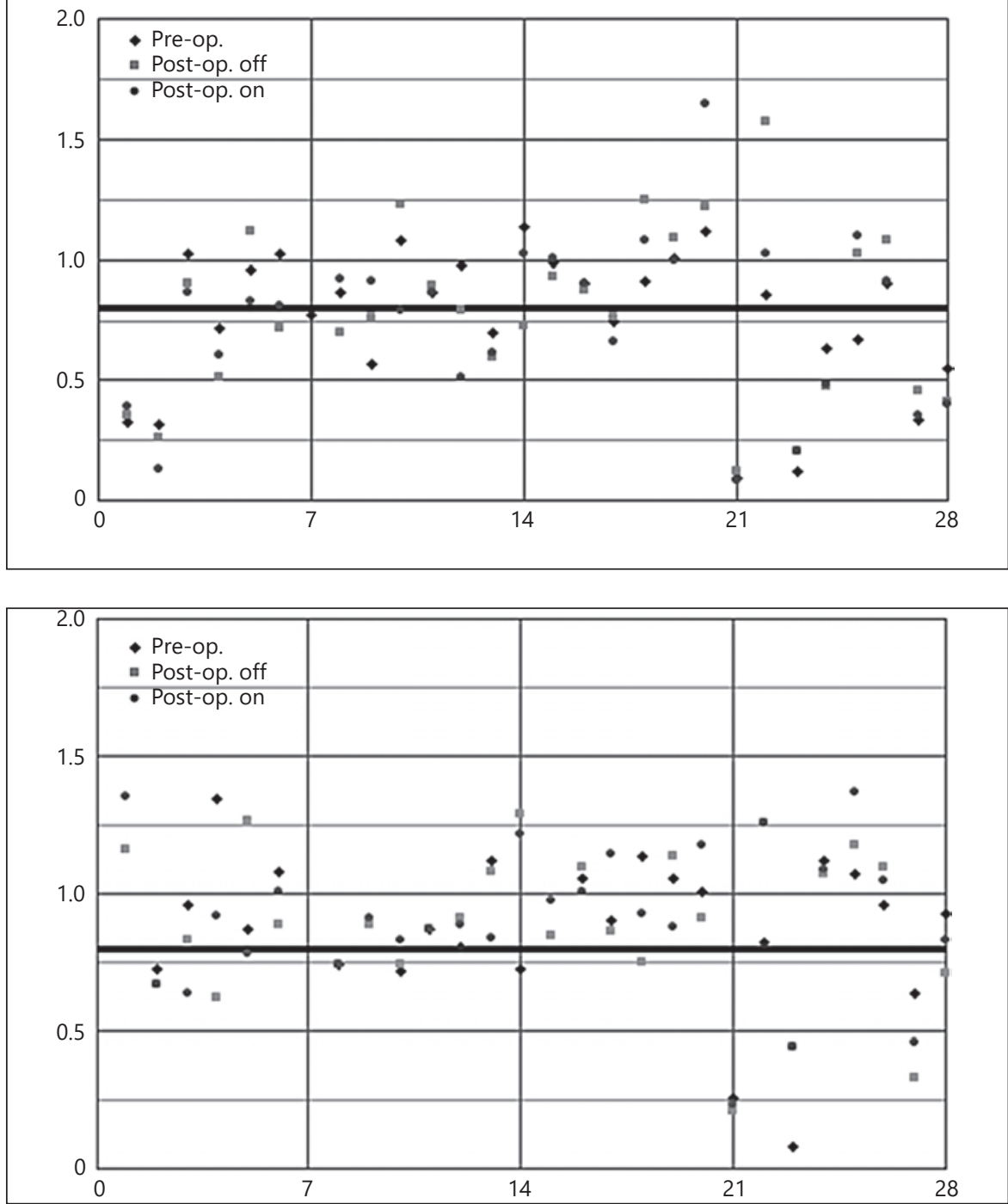

\section{Implanted Side}

- LSC vHIT (all 28 subjects): the VOR gain was found to be reduced $[<0.8$ according to Alhabib and Saliba, 2017] in 13 subjects $(46.4 \%)$ and normal $(>0.8)$ in the remaining subjects ( $n=15 ; 53.6 \%$; Fig. 1$)$.

- SSC vHIT (24 out of 28 subjects): the VOR gain was found to be reduced $(<0.8)$ in 7 subjects $(29.2 \%)$ and normal $(>0.8)$ in the remaining subjects $(n=17 ; 70.8 \%$; Fig. 2).

- PSC vHIT ( 24 out of 28 subjects): the VOR gain was found to be reduced $(<0.8)$ in 6 subjects $(25 \%)$ and nor$\mathrm{mal}(>0.8)$ in the remaining subjects $(n=18,75 \%$; Fig. 3$)$.

- cVEMP (23 out of 28 subjects): they were present and normal in 5 subjects $(21.7 \%)$ and absent in the remaining subjects ( $n=17,73.9 \%$; Table 2$)$.

\section{Nonimplanted Side}

- LSC vHIT (all 28 subjects): the VOR gain was found to be reduced $(<0.8)$ in 11 subjects $(39.3 \%)$ and normal $(>0.8)$ in the remaining subjects ( $n=17 ; 60.7 \%$; Fig. 1$)$.

- SSC vHIT (24 out of 28 subjects): the VOR gain was found to be reduced $(<0.8)$ in 7 subjects $(29.2 \%)$ and normal $(>0.8)$ in the remaining subjects $(n=17 ; 70.8 \%$; Fig. 2).

- PSC vHIT (24 out of 28 subjects): the VOR gain was found to be reduced $(<0.8)$ in 8 subjects $(33.3 \%)$ and normal $(>0.8)$ in the remaining subjects $(n=16 ; 66.7 \%$; Table 4).

- cVEMP (15 out of 28 subjects): they were present and normal in 8 subjects (53.3\%) and absent in the remaining 7 subjects ( $n=7 ; 46.7 \%$; Table 2$)$. 
Fig. 3. vHIT for the PSC in preoperative and postoperative conditions (in off and on situations).

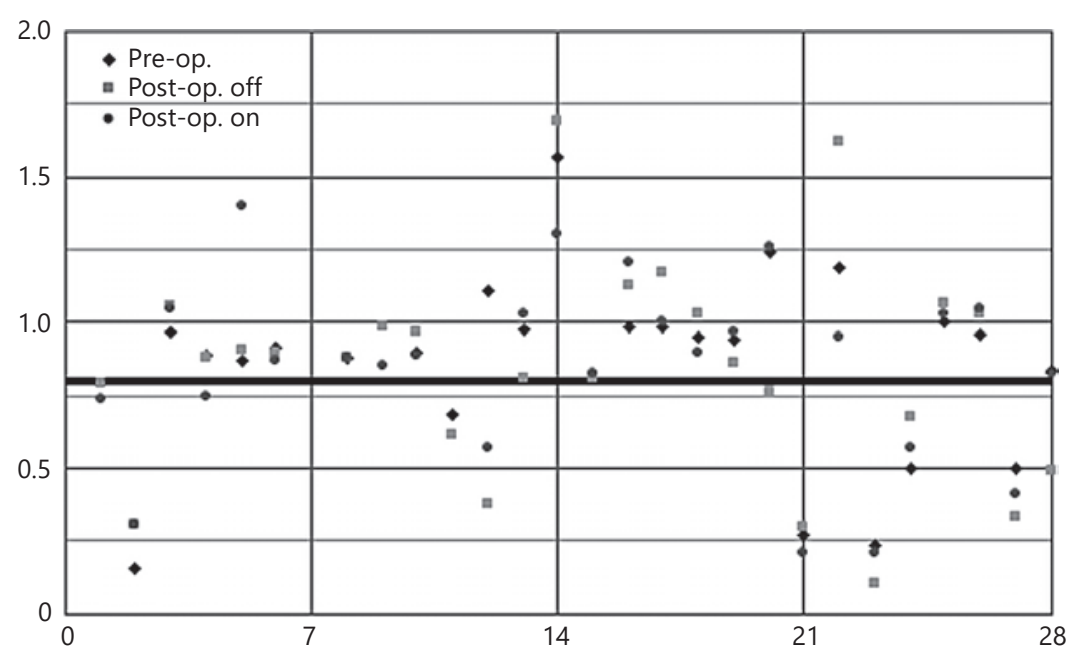

At CI activation with the implant off, the findings were as follows.

\section{Implanted Side}

- LSC vHIT (27 out of 28 subjects): the VOR gain was found to be reduced $(<0.8)$ in 15 subjects $(55.6 \%)$ and normal $(>0.8)$ in the remaining subjects $(n=12 ; 44.4 \%$; Fig. 1).

- SSC vHIT (27 out of 28 subjects): the VOR gain was found to be reduced $(<0.8)$ in 9 subjects $(33.3 \%)$ and normal $(>0.8)$ in the remaining subjects $(n=18 ; 66.7 \%$; Fig. 2).

- PSC vHIT (27 out of 28 subjects): the VOR gain was found to be reduced $(<0.8)$ in 10 subjects $(37 \%)$ and nor$\mathrm{mal}(>0.8)$ in the remaining subjects $(17 ; 63 \%$; Fig. 3$)$.

\section{Nonimplanted Side}

- LSC vHIT (27 out of 28 subjects): the VOR gain was found to be reduced $(<0.8)$ in 12 subjects $(44 \%)$ and normal $(>0.8)$ in the remaining subjects $(n=15 ; 55.6 \%$; Fig. 1).

- SSC vHIT (27 out of 28 subjects): the VOR gain was found to be reduced $(<0.8)$ in 6 subjects $(22.2 \%)$ and normal $(>0.8)$ in the remaining subjects $(n=21 ; 67.8 \%$; Fig. 2).

- PSC vHIT (27 out of 28 subjects): the VOR gain was found to be reduced $(<0.8)$ in 8 subjects $(29.6 \%)$ and normal $(>0.8)$ in the remaining subjects $(n=19 ; 70.4 \%$; Fig. 3).

- cVEMP ( 6 out of 28 subjects): they were present in 5 subjects $(83.3 \%)$ and absent in the remaining subject (16.7\%; Table 2).
At $\mathrm{CI}$ activation, with the implant on, the findings were as follows.

\section{Implanted Side}

- LSC vHIT (27 out of 28 subjects): the VOR gain was found to be reduced $(<0.8)$ in 12 subjects $(44.4 \%)$ and normal $(>0.8)$ in the remaining subjects $(n=15 ; 55.6 \%$; Fig. 1).

- SSC vHIT (27/28 subjects): the VOR gain was found to be reduced $(<0.8)$ in 7 subjects $(25.9 \%)$ and normal $(>0.8)$ in the remaining subjects ( $n=20 ; 74.1 \%$; Fig. 2$)$.

- PSC vHIT (27 out of 28 subjects): the VOR gain was found to be reduced $(<0.8)$ in 9 subjects $(33.3 \%)$ and normal $(>0.8)$ in the remaining subjects $(n=18 ; 66.7 \%$; Fig. 3$)$.

- cVEMP ( 6 out of 28 subjects): they were absent in all of the tested subjects (100\%; Table 2$)$.

\section{Nonimplanted Side}

- LSC vHIT (27 out of 28 subjects): the VOR gain was found to be reduced $(<0.8)$ in 12 subjects $(40.7 \%)$ and normal $(>0.8)$ in the remaining subjects $(n=16 ; 59.3 \%$; Fig. 1).

- SSC vHIT (27 out of 28 subjects): the VOR gain was found to be reduced $(<0.8)$ in 7 subjects $(25.9 \%)$ and normal $(>0.8)$ in the remaining subjects $(n=20 ; 74.1 \%$; Fig. 2).

- PSC vHIT (27 out of 28 subjects): the VOR gain was found to be reduced $(<0.8)$ in 9 subjects $(33.3 \%)$ and normal $(>0.8)$ in the remaining subjects $(n=18 ; 66.7 \%$; Fig. 3$)$.

- cVEMP (6 out of 28 subjects): they were present and normal in 5 subjects (83.3\%) and absent in the remaining subject (16.7\%; Table 2). 
Table 2. Pre- and postoperative vestibular assessment in the study group, including cVEMP of the implanted (CI) and non-implanted ears, DHI questionnaire scores, and the presence of vertigo

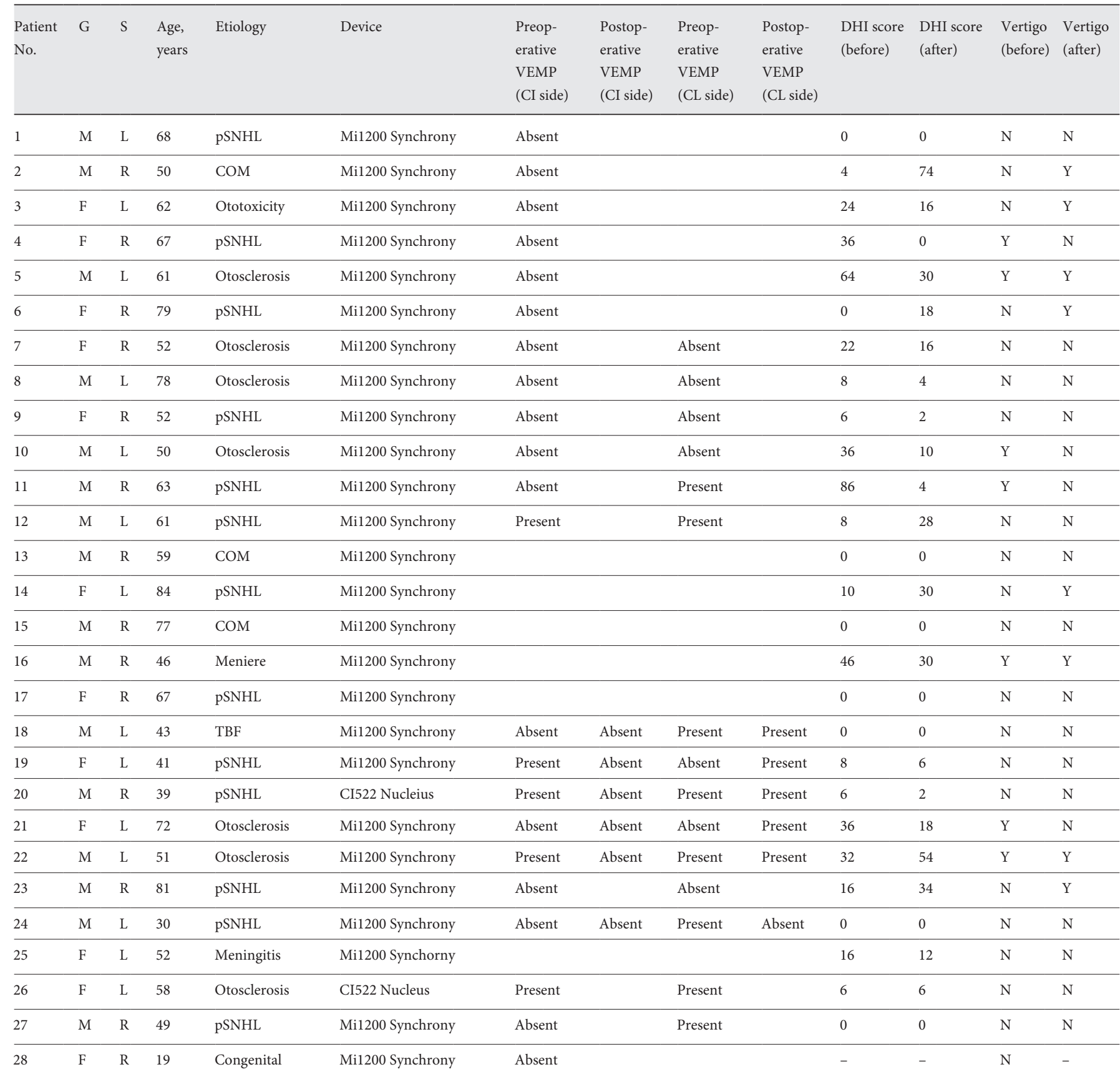

pSNHL, progressive sensorineural hearing loss; COM, chronic otitis media; TBF, temporal bone fracture. G, gender; M, male; F, female; S, side of surgery; R, right; L, left; CL, contralateral, nonimplanted ear; $\mathrm{Y}$, yes, $\mathrm{N}$, no.

The postoperative DHI score showed increased impairment in 7 subjects $(25 \%)$, with a mean difference of 48 (range 18-70; Table 2).

Specific data regarding special categories of patients can be observed.
- In the operated side, the vHIT of the LSC yielded better evidence of a reduced vestibular function in comparison to the other 2 SSC. Likewise, the CVEMP indicated that nearly $80 \%$ of the vestibular impairment was at the level of the saccule. In $50 \%$ of the subjects with 
Table 3. vHIT in the otosclerotic group

\begin{tabular}{|c|c|c|c|c|c|c|c|c|c|c|c|c|c|c|c|c|c|c|c|}
\hline \multirow{2}{*}{$\begin{array}{l}\text { Patient } \\
\text { No. }\end{array}$} & \multirow{2}{*}{ Approach } & \multicolumn{3}{|c|}{ Pre-vHIT (CI side) } & \multicolumn{3}{|c|}{ Post-vHIT (CI off) } & \multicolumn{3}{|c|}{ Post-vHIT (CI on) } & \multicolumn{3}{|c|}{ Pre-vHIT (CL side) } & \multicolumn{3}{|c|}{ Post-vHIT (CL off) } & \multicolumn{3}{|c|}{ Post-vHIT (CL on) } \\
\hline & & LSC & SSC & PSC & LSC & SSC & PSC & LSC & SSC & PSC & LSC & SSC & PSC & LSC & SSC & PSC & LSC & SSC & PSC \\
\hline 1 & eRWA & 0.63 & 1.12 & 0.50 & 0.48 & 1.07 & 0.68 & 0.49 & 1.09 & 0.57 & 0.88 & 1.02 & 0.86 & 0.77 & 1 & 0.87 & 0.74 & 0.89 & 0.84 \\
\hline 2 & eRWA & 0.87 & 0.74 & 0.88 & 0.7 & 0.74 & 0.88 & 0.92 & 0.74 & 0.88 & 0.73 & 0.91 & 0.96 & 0.56 & 0.91 & 0.96 & 0.69 & 0.91 & 0.96 \\
\hline 3 & eRWA & 0.57 & & & 0.76 & & & 0.91 & & & 0.88 & & & 0.96 & & & 0.74 & & \\
\hline 4 & RWA & 0.72 & 1.35 & 0.89 & 0.51 & 0.62 & 0.88 & 0.61 & 0.92 & 0.75 & 0.91 & 0.63 & 1.24 & & & & & & \\
\hline 5 & RWA & 1.02 & 1.08 & 0.92 & 0.72 & 0.89 & 0.9 & 0.81 & 1.01 & 0.87 & 1.09 & 1.1 & 0.93 & 0.98 & 0.85 & 0.91 & 1.08 & 1.04 & 0.9 \\
\hline 6 & RWA & 1.02 & 1.08 & 0.97 & 0.9 & 1.06 & 0.83 & 0.87 & 1.05 & 0.64 & 1.06 & 1.01 & 1.13 & 0.93 & 0.58 & 0.7 & 0.91 & 0.88 & 0.88 \\
\hline 7 & RWA & 0.55 & 0.93 & 0.84 & & & & & & & 0.56 & 0.88 & 0.85 & & & & & & \\
\hline
\end{tabular}

CL, contralateral, nonimplanted ear.

absent cVEMP, an associated reduced VOR gain was found. Similar data were also found for the nonoperated ear ( $~ 60 \%$ of the patients showed a reduced VOR gain of the LSC in the nonoperated ear), meaning that the basal pathology was similarly affecting both ears.

- Four subjects with different etiologies (progressive sensorineural hearing loss [SNHL], chronic otitis media, ototoxicity, and meningitis) presented a preoperative low VOR gain in both implanted and nonimplanted ears, and for each SSC investigated that persisted at the postoperative assessment. Likewise, in these subjects, cVEMP were absent pre- and postoperatively.

- In the otosclerosis group $(n=7)$, both abnormal ( $n=$ $4)$ and normal $(n=3)$ VOR gains of the LSC were found preoperatively and in only 1 of the 4 abnormal subjects did the VOR gain normalize postoperatively (Table 3). Within this group there was also the most likely situation (42.8\% of the study group) for performing an eRWA instead of the planned RWA.

- The Ménière subject, also affected by drop attack episodes, presented normal VOR gain values that were not affected by surgery. Interestingly, no further episodes of DA occurred up to 2 years after CI, as also shown by the DHI score (from 46 to 30 ).

- In the patient with partial deafness (progressive SNHL) in whom the soft-surgery protocol was applied, a bilateral decreased VOR gain was found in the preoperative assessment. It was found to have returned to normality on the postoperative tests, with the implant off and on.

- In the patient who had already received CI in the opposite ear, a low VOR gain in the LSC of the first operated ear was present at all evaluation times. In the ear implanted for this study, a normal VOR gain was preoperatively found with a decrease at CI activation/implant off and renormalization with the implant on.

No significant differences in the mean gain values between preoperative time, postoperative time (implant off), and post-operative time (implant on) were detected $(p=0.399)$. Patients with complete data $(n=6)$ were included in the statistical analysis of the cVEMPS. No statistically significant difference was reported between the distributions of the different combinations of the analyzed series $(p \geq 0.083)$. No significant differences in DHI score changes were detected between the preoperative and postoperative means $(p=0.658)$.

\section{Discussion}

The reports of the effects of CI on vestibular function are controversial and several factors have been claimed to support a negative influence of this rehabilitative surgical procedure. In this regard, intraoperative loss of perilymph [Mangham, 1987], postcochleostomy labyrinthitis and endolymphatic hydrops [Fina et al., 2003], electrical stimulation [Bance et al., 1998], foreign body-induced labyrinthitis [Kubo et al., 2001], and direct injury [O'Leary et al., 1991] have been indicated. Overall, the environment in common and the anatomical proximity of the cochlear and vestibular structures are likely to suggest a mutual interaction both in physiological and in pathological situations. In particular, the saccule and the lateral SSC have been considered the vestibular organs that are more likely to be endangered by CI [Handzel et al., 2006].

Based on this premise, the use of investigational tools that can provide useful data for testing the vestibular function is of the utmost importance. A modern ap- 
Table 4. Cervical vestibular evoked myogenic potentials in the otosclerotic group

\begin{tabular}{|c|c|c|c|c|c|c|c|}
\hline $\begin{array}{l}\text { Patient } \\
\text { No. }\end{array}$ & Approach & $\begin{array}{l}\text { Pre-AC cVEMP } \\
\text { (CI side) }\end{array}$ & $\begin{array}{l}\text { Post-AC cVEMPs } \\
\text { (CI side) }\end{array}$ & $\begin{array}{l}\text { Pre-AC cVEMPs } \\
\text { (CL side) }\end{array}$ & $\begin{array}{l}\text { Post-AC cVEMP } \\
\text { (CL side) }\end{array}$ & $\begin{array}{l}\text { Preoperative } \\
\text { vertigo }\end{array}$ & $\begin{array}{l}\text { Postoperative } \\
\text { vertigo }\end{array}$ \\
\hline 1 & eRWA & A & A & A & A & $\mathrm{P}$ & $\mathrm{P}$ \\
\hline 3 & eRWA & A & A & A & A & A & A \\
\hline 4 & RWA & $\mathrm{A}$ & A & $\mathrm{A}$ & A & $\mathrm{P}$ & $\mathrm{A}$ \\
\hline 5 & RWA & $\mathrm{A}$ & $\mathrm{A}$ & A & $\mathrm{P}$ & $\mathrm{P}$ & A \\
\hline 7 & RWA & $\mathrm{P}$ & A & $\mathrm{P}$ & $\mathrm{A}$ & A & $\mathrm{A}$ \\
\hline
\end{tabular}

CL, contralateral, non-implanted ear; P, present; A, absent.

proach relies on objective procedures that may enable the professional to gain evidence, singularly, of the actual condition of the entire posterior labyrinth, i.e., the vHIT for investigating the VOR of each SSC, and the VEMP for investigating the macular structures (utricle and saccule). Previous reports have considered both caloric and rotatory chair testing as the most appropriate for vestibular assessment [Rah et al., 2016; Bittar et al., 2019]. However, using vHIT and VEMP one would expect a more precise definition of parcellar impairment, being moreover more adherent to a physiological condition since the caloric (nonphysiological) stimulus and the rotatory chair are probing the low- to mid-frequency band of the VOR response $(0.1-1 \mathrm{~Hz})$, leaving untested the higher-frequency $(1-16 \mathrm{~Hz})$ components of head movement that are responsible for gaze stabilization [Grossman et al., 1988].

When considering the possible influence of $\mathrm{CI}$ on the vestibular system, it is reasonable to take into account several variables, including the possibility that the pathology causing deafness (bilaterally) might also present with associated vertigo/balance impairment. This aspect could be investigated by testing both the implanted ear and the contralateral, nonimplanted ear preoperatively, along with a thorough history taking and specific questionnaires which were, in fact, routinely performed in the present study. The preoperative assessment of our study group revealed a bilateral reduced VOR gain of the LSC only in $<50 \%$ of the subjects affected by progressive SNHL $(n=7)$, otosclerosis $(n=2)$, congenital $(n=1)$, meningitis $(n=1)$, chronic otitis $(n=1)$, and ototoxicity $(n=1)$, while it involved all the 3 superior SSC in only 4 of them (1 with meningitis, 1 with ototoxicity, 1 with otosclerosis, and 1 with chronic otitis). Interestingly, in the latter group this finding was associated with the absence of cVEMP as a proof of saccular involvement [Curthoys et al., 2016]. The absence of cVEMP could also be attributed to the hearing loss that would affect the air conduction stimulation that is generally used [Papathanasiou et al., 2014]. This latter finding, however, is not in accordance with what has recently been reported [Rosengren et al., 2019], i.e., that while a conductive hearing loss may impair VEMP results, an SNHL including total deafness should not be influential, although in specific pathologies like chronic otitis media and otosclerosis, represented in the current study group, one may likely expect the concomitant presence of an important conductive component for the hearing loss. In addition, the postoperative VOR data of the LSC in the hypofunctional group indicate an unchanged pattern in 10 patients with the implant off and on and an improved pattern in 1 subject with the implant on and in 2 subjects with the implant off and on. Surprisingly, in the only Ménière patient (with drop attacks) in this study, normal VOR gain values were recorded both preoperatively and postoperatively, with an evident decrease in the DHI score after surgery as witnessed by the absence of further drop attack episodes.

The possible influence of a direct labyrinthine injury [O'Leary et al., 1991] was investigated in our study group, which was composed of adults who underwent (except in 1 case) CI on one side only. In this situation, one may assume that the selection of the ear to be implanted should also take into consideration differences in vestibular function between the 2, thus taking the worst vestibular functioning ear as a further variable to consider, especially if the hearing condition is similar between the 2 ears. In this scenario, in fact, it is possible to hypothesize that by implanting the ear with poorer vestibular function less direct damage could be expected, also enabling the better contralateral ear for an appropriate central com- 
pensatory mechanism to be elicited. In case of a simultaneous (or sequential) bilateral implantation, such as is routinely the case in the pediatric population, it is possible to speculate on different mechanisms that could affect the vestibular organs. Since the present study did not take into account $\mathrm{CI}$ in children, this aspect was investigated.

The surgical procedure for electrode placement is considered among the possible factors affecting the vestibular function [Usami et al., 2011]. Theoretically, a soft-surgery procedure could somewhat warrant the least trauma also to the vestibular organs, with some reports supporting this theory [Adunka et al., 2004; Tsukada et al., 2013; Sosna et al., 2019] and others considering it not influential [Korsager et al., 2018]. In the present study, in the only subject with partial deafness in whom an EAS was planned, implementing the protocol with systemic and local steroids, the postoperative evaluation revealed a persistence of normal parameters. These findings were also discovered when using cVEMP, which are considered the best diagnostic tool for exploring the function of the saccule, with the latter being described as the vestibular end organ at major risk due to its closeness to the cochlear site that is violated when performing CI [Tien and Linthicum, 2002]. In this regard, Melvin et al. [2009] reported cVEMP evidence of saccular injury in $31 \%$ of implanted ears. By choosing the RWA, moreover, the surgeon is potentially attempting to prevent the whole inner ear compartment from being damaged by the possible migration of the electrode carrier from the scala tympani to the scala vestibuli through the scala media and spiral lamina, as has been described to occur with a higher incidence when a standard cochleostomy is performed [Fischer et al., 2015; Jiam and Limb, 2016]. In this regard, Todt and colleagues reported $50 \%$ of postoperative absent cVEMP in patients who underwent cochleostomy but only in 13\% of the RWA approach group [Basta et al., 2008]. One may also suggest that the disappearance of cVEMP postoperatively could be due to the presence of the electrode array that would prevent less acoustic energy from being delivered to the cochlea while, considering the very short-term analysis of the present study (1 month postoperatively), the development of fibrosis would be less likely to be influential.

In spite of nonsignificant differences when testing the vestibular system with vHIT or CVEMP, subjectively the study group showed improvement at DHI (from 17.4 to 14.6), similar to what has been previously reported [Shoman et al., 2008; Zawawi et al., 2014].

Although CI encompasses violation of the bony or membranous inner ear structures, the present study re- vealed no changes in vestibular function with respect to the preoperative situation. Although the short time chosen for vestibular assessment could be considered a limiting factor for an appropriate vestibular evaluation after CI, it has offered some interesting results that logically only include the causative pathology and the surgical procedure, while other factors such as electrical stimulation or postoperative fibrosis that would occur at a later stage cannot be taken into consideration. Whether these latter factors would somehow be influential or not can only be assessed by following up the same study group for a longer period.

\section{Acknowledgement}

The authors are thankful to the engineer Mr. Daniele Ferrante for helping with the statistical evaluation of the data.

\section{Statement of Ethics}

The subjects gave their written informed consent and the study protocol was approved by the local Ethical Committee on Human Research at the Sant'Andrea University Hospital, Rome, Italy, and was performed in accordance with the ethical guidelines of the 1975 Declaration of Helsinki.

\section{Disclosure Statement}

The authors have no conflict of interests to declare.

\section{Funding Sources}

No institutional or private funding was obtained for the present research.

\section{Author Contributions}

M.B. designed this work, revised the intellectual content, gave final approval of the version to be published, and agreed to be accountable for all aspects of this work. R.T. and A.T.B. analyzed the data, drafted this work, gave final approval of the version to be published, and agreed to be accountable for all aspects of this work. S.T. and C.F. acquired data, drafted this work, gave final approval of the version to be published, and agreed to be accountable for all aspects of this work. E.C. designed this work; drafted the work, gave final approval of the version to be published, and agreed to be accountable for all aspects of this work. S.M. interpreted the data, revised the intellectual content, gave final approval of the version to be published, and agreed to be accountable for all aspects of this work.
Barbara/Talamonti/Benincasa/Tarentini/ Filippi/Covelli/Monini 


\section{References}

Adunka O, Unkelbach MH, Mack M, Hambek M, Gstoettner W, Kiefer J. Cochlear implantation via the round window membrane minimizes trauma to cochlear structures: a histologically controlled insertion study. Acta Otolaryngol. 2004 Sep;124(7):807-12.

Alhabib SF, Saliba I. Video head impulse test: a review of the literature. Eur Arch Otorhinolaryngol. 2017 Mar;274(3):1215-22.

Bance ML, O'Driscoll M, Giles E, Ramsden RT. Vestibular stimulation by multichannel cochlear implants. Laryngoscope. 1998 Feb; 108(2):291-4.

Basta D, Todt I, Goepel F, Ernst A. Loss of saccular function after cochlear implantation: the diagnostic impact of intracochlear electrically elicited vestibular evoked myogenic potentials. Audiol Neurotol. 2008;13(3):187-92.

Batuecas-Caletrio A, Klumpp M, Santacruz-Ruiz S, Benito Gonzalez F, Gonzalez Sánchez E, Arriaga M. Vestibular function in cochlear implantation: correlating objectiveness and subjectiveness. Laryngoscope. 2015 Oct; 125(10):2371-5.

Bittar RS, Sato ES, Silva-Ribeiro DJ, Oiticica J, Mezzalira R, Tsuji RK, et al. Caloric test and video head impulse test sensitivity as vestibular impairment predictors before cochlear implant surgery. Clinics (São Paulo). 2019 Mar; 74:e786.

Black FO. Present vestibular status of subjects implanted with auditory prostheses. Ann Otol Rhinol Laryngol 1977;Suppl 38:49-56.

Buchman CA, Joy J, Hodges A, Telischi FF, Balkany TJ. Vestibular effects of cochlear implantation. Laryngoscope. 2004 Oct;114(10 Pt 2 Suppl 103):1-22.

Coordes A, Basta D, Gotze R, Scholz S, Seidl RO, Ernst A, Todt I. Sound-induced vertigo after cochlear implantation. Otol Neurotol 2012; 33:335-42.

Curthoys IS, Vulovic V, Burgess AM, Sokolic L, Goonetilleke SC. The response of guinea pig primary utricular and saccular irregular neurons to bone-conducted vibration (BCV) and air-conducted sound (ACS). Hear Res. 2016 Jan;331:131-43.

Eisenberg LS, Nelson JR, House WF. Effects of the single-electrode cochlear implant on the vestibular system of the profoundly deaf adult. Ann Otol Rhinol Laryngol Suppl. 1982 MarApr;91(2 Pt 3):47-54.

Fina M, Skinner M, Goebel JA, Piccirillo JF, Neely JG, Black O. Vestibular dysfunction after cochlear implantation. Otol Neurotol. 2003 Mar;24(2):234-42.
Fischer N, Pinggera L, Weichbold V, Dejaco D, Schmutzhard J, Widmann G. Radiologic and functional evaluation of electrode dislocation from the scala tympani to the scala vestibuli in patients with cochlear implants. AJNR Am J Neuroradiol. 2015 Feb;36(2):372-7.

Grossman GE, Leigh RJ, Abel LA, Lanska DJ, Thurston SE. Frequency and velocity of rotational head perturbations during locomotion. Exp Brain Res. 1988;70(3):470-6.

Handzel O, Burgess BJ, Nadol JB Jr. Histopathology of the peripheral vestibular system after cochlear implantation in the human. Otol Neurotol. 2006 Jan;27(1):57-64.

Jiam NT, Limb CJ. The impact of round window vs cochleostomy surgical approaches on interscalar excursions in the cochlea: Preliminary results from a flat-panel computed tomography study. World J Otolaryngol Head Neck Surg; 2016. pp. 1-6.

Jin Y, Shinjo Y, Akamatsu Y, Ogata E, Nakamura M, Kianoush S, Yamasoba T, Kaga K. Vestibular evoked myogenic potentials evoked by multichannel cochlear implant influence of C levels. Acta Otolaryngol. 2008;128:284-90.

Korsager LE, Schmidt JH, Faber C, Wanscher JH. Vestibular Outcome After Cochlear Implantation Is Not Related to Surgical Technique: A Double Blinded, Randomized Clinical Trial of Round Window Approach Versus Cochleostomy. Otol Neurotol. 2018 Mar;39(3):30612.

Kubo T, Yamamoto K, Iwaki T, Doi K, Tamura M. Different forms of dizziness occurring after cochlear implant. Eur Arch Otorhinolaryngol. 2001 Jan;258(1):9-12.

Mangham CA. Effects of cochlear prostheses on vestibulo-ocular reflexes to rotation. Ann Otol Rhinol Laryngol Suppl. 1987;96(1_suppl):101-4.

Melvin TA, Della Santina CC, Carey JP, Migliaccio AA. The effects of cochlear implantation on vestibular function. Otol Neurotol. 2009 Jan;30(1):87-94.

Nola G, Mostardini C, Salvi V, Ercolano AP, Ralli G. Validity of Italian adaptation of the Dizziness Handicap Inventory (DHI) and evaluation of the quality of life in patients with acute dizziness. Acta Otorhinolaryngol Ital. 2010 Aug;30(4):190.

O'Leary MJ, Fayad J, House WF, Linthicum FH Jr. Electrode insertion trauma in cochlear implantation. Ann Otol Rhinol Laryngol. 1991 Sep;100(9 Pt 1):695-9.
Papathanasiou ES, Murofushi T, Akin FW, Colebatch JG. International guidelines for the clinical application of cervical vestibular evoked myogenic potentials: an expert consensus report. Clin Neurophysiol. 2014 Apr;125(4): 658-66.

Parkes WJ, Gnanasegaram JJ, Cushing SL, McKnight CL, Papsin BC, Gordon KA. Vestibular evoked myogenic potential testing as an objective measure of vestibular stimulation with cochlear implants. Laryngoscope 2017;127: E75-E81.

Rah YC, Park JH, Park JH, Choi BY, Koo JW. Dizziness and vestibular function before and after cochlear implantation. Eur Arch Otorhinolaryngol. 2016 Nov;273(11):3615-21.

Roland PS, Wright CG, Isaacson B. Cochlear implant electrode insertion: the round window revisited. Laryngoscope. 2007 Aug;117(8): 1397-402.

Rosengren SM, Colebatch JG, Young AS, Govender S, Welgampola MS. Vestibular evoked myogenic potentials in practice: Methods, pitfalls and clinical applications. Clin Neurophysiol Pract. 2019 Feb;4:47-68.

Shoman N, Ngo R, Archibald J, Pijl S, Chan S, Westerberg BD. Prevalence of new-onset vestibular symptoms following cochlear implantation. J Otolaryngol Head Neck Surg. 2008 Jun;37(3):388-94.

Sosna M, Tacikowska G, Pietrasik K, Skarżyński H, Lorens A, Skarżyński PH. Effect on vestibular function of cochlear implantation by partial deafness treatment-electro acoustic stimulation (PDT-EAS). Eur Arch Otorhinolaryngol. 2019 Jul;276(7):1951-9.

Tien HC, Linthicum FH Jr. Histopathologic changes in the vestibule after cochlear implantation. Otolaryngol Head Neck Surg. 2002 Oct;127(4):260-4.

Tsukada K, Moteki H, Fukuoka H, Iwasaki S, Usami S. Effects of EAS cochlear implantation surgery on vestibular function. Acta Otolaryngol. 2013 Nov;133(11):1128-32.

Usami S, Moteki H, Suzuki N, Fukuoka H, Miyagawa M, Nishio SY, et al. Achievement of hearing preservation in the presence of an electrode covering the residual hearing region. Acta Otolaryngol. 2011 Apr;131(4): 405-12.

Zawawi F, Alobaid F, Leroux T, Zeitouni AG. Patients reported outcome post-cochlear implantation: how severe is their dizziness? J Otolaryngol Head Neck Surg. 2014 Dec;43(1): 49. 